\title{
APORTACION AL CONOCIMIENTO DE LOS HONGOS DEL S.E. DE ESPAÑA. I
}

\author{
M. HONRUBIA \& X. LLIMONA *
}

\section{RESUMEN:}

Primer inventario de hongos de la región murciana, observados en otono de 1977. Figuran en él especies recogidas en la huerta, en jardines, dunas, pinares instalados sobre todo en el área potencial del Chamaeropo-Rhamnetum lycioidis y del Quercetum rotundifoliae, hasta el límite del quejigar (en la Sierra de Caravaca).

Se comenta el cortejo floristico hallado en cada tipo de ambiente. Entre las especies mediterráneas interesantes pueden destacarse: Ciriporia bresadolae, sobre madera dura de pino, Boletopsisleucomelas, Camarophyllus carneogriseus, Tricholomacaligatum, T. psammopus, Cortinarius ionochlorus, Geastrum pseudostriatum.

\section{SUMMARY:}

A preliminary list of fungi collected in late autumn of 1977 in the region of Murcia (SE Spain), including species found in orchards and parks, dunes, clear pine woods in the poten tial area of Chamatropo-Rhamnetum lycioidis and in the potential area of Quercetum rotundifo liate, to the same limit of the area of Quercus valentina (in the Sierra de Caravaca).

For each ecological residence studied, typical ensemble of species is commented. Some interesting mediterranean species collected are: Ceriporia bresadolat, on hard pine wood, $\dot{B}$ oletopsis leucomelas, Camarophyllus carneogrisies, Tricholoma cadigatum. T. psammopus, Cortinarius ionocblorus, Geastrum pieudostriatum.

Debido a la escasez y, sobre todo, a la irregularidad de las lluvias, el inventario de la flora fúngica de una región como la murciana se convierte en una tarea difícil y discontinua. Sólo contando con un buen grupo de recolectores y una buena información sobre los puntos en los que ha llovido y, principalmente, residiendo en el propio territorio, se hace posible efectuar, si las temporadas son favorables, recolecciones de carpóforos que den idea de la actividad miceliar (la mayor parte del año aletargada por falta de agua). Sólo así será posible, acumulando los datos de varios años, hacerse cargo de la importancia de los hongos en los ecosistemas terestres de la región. 
La creación de un departamento de Botánica en la Universidad de Murcia, y la circunstancia de un otoño (el de 1977), bastante lluvioso para la región, junto con la colaboración de profesores (J.M. Egea, R. Verdú, G. López Vélez), de numerosos alumnos y de personal de ICONA, a los que expresamos nuestro agradecimiento, han hecho posible esta primera visión, muy parcial, de la flora fúngica murciana.

Las lluvias a que nos referimos cayeron a finales de Octubre, y sobre todo a finales de Noviembre. Se visitaron personalmente algunas localidades como la Sierra de la Fuensanta (29/X/77), Sierra de Caravaca, alrededores del Nevazo (31/X), dunas con pinar de San Pedro del Pinatar (5/XI), Sierra de Espuña (3/XII).

A excepción de algunas especies muy conocidas, el material citado en este trabajo se conserva en el herbario del Departamento (MURCIA Micoteca). Se conserva también una colección de diapositivas con el aspecto en fresco de las recolecciones más interesantes.

Una parte de lo recogido no viene incluida en este trabajo por hallarse inidentificable o pendiente de confirmación. Una de las consecuencias de ello es que la riqueza en Agaricus de la huerta murciana no aparece reflejada en este trabajo.

Por otro lado, los mixomicetes recolectados, sorprendentemente numerosos. (p. ej. los que pululan bajo los cladodios muertos de las chumberas), serán objeto de un trabajo independiente (E. Gracia, en prensa).

La impresión que se desprendería de este primer contacto, sin duda muy incompleto, es la de una flora más bien pobre en especies de agaricales sobre todo en las tierras bajas y áridas.

Los pinares xéricos, de las vertientes bajas de las sierras de Carrascoy, Cartagena, Espuña y parte basal de otras sierras próximas presentan, después de un período de lluvias intensas, una abundante floración fúngica, dominada por Suillus flur$y i,(=$ collinitus) del grupo granulatus, a menudo acompañado por Suillus bellinit, Chroogomphus rutilus y Amanita ovoidea, que caracterizan la flora xérica y termófila que explota la gruesas alfombras de agujas de pino que se acumulan durante los largos períodos de sequía. Al mismo tiempo establecen micorrizas con Pinus halepensis. Sólo esta doble disponibilidad de alimentos puede explicar la enorme (y fugaz) biomasa de carpóforos de Suillus y Amanita que aparecen en los otoños favorables. Hemos visto casos semejantes en Valencia (p. ej. en Fuente La Higuera) y en Ibiza y Formentera (navidad de 1967).

Las ramas muertas de Pinus halepensis sufren sobre el suelo una descomposición lenta, debido a los largos períodos secos, y conservan largo tiempo una gran dureza. Constituyen el sustrato de elección de una característica poria, hasta ahora muy poco citada, Ceriporia bresadolae, una de las especies más abundantes y características de los pinares térmicos murcianos.

Sobre las dunas litorales, (p. ej. en San Pedro del Pinatar), se establecen pinares que presentan, junto a nutridas poblaciones de los Suillus ya citados, verdaderas cohortes de un Inocybe del grupo dulcamara, que asimilamos a I.caesariata, ya conocido de otros pinares litorales como los de Castelldefels (Heim 1931, Losa 1960).

A pesar de ser buenos comestibles, los dos Suillus en cuestión, son despreciados por los micófagos locales que los denominan despectivamente "bojines". Es cierto que son pronto pasto de larvas o sufren la invasión de la forma conídica Sepedonium, del ascomicete Apiocraea chrysosperma, que las deforma y envuelve en un manto de clamidósporas doradas. 
A mayor altitud, los pinares menos secos se enriquecen en los codiciados guíscanos (nombre local): Lactarius deliciosus y L. sanguifluus. Le acompaña un cortejo de especies asociadas al pino, entre las que hemos visto: Tricholoma ustaloides, T. caligatum, T. psammopus, T. terreum, Rhizopogon rubescens, Hebeloma edurum, Collybia dryophila. La aparición demasiado tardía de las lluvias, no permitió observar otras Russula que $R$. torulosa y $R$. sanguinea.

La madera muerta de los pinos es destruída lentamente por especies como Dacrymyces deliquescens, muy abundante, persistente en tiempo seco en forma de gránulos córneos negruzcos, Schizophyllum commune, Ruststroemia firma, sobre ramas; mientras que los troncos aún vivos sufren el ataque de Phellinus pini y los tocones son degradados por Stereum birsutum, Paxillus panuoides y Fomitopsis pinicola, en dura competencia con los coleópteros xilófagos.

Los árboles de ribera son en cambio pasto preferido de Inonotus hispidus, que puede alcanzar enormes tamaños sobre los viejos chopos. Los tronnes de estos árboles suelen presentar asimismo, los agradables Agrocybe aegerita y Pleurotus o:treatus, junto a Stereum purpureum y Flammulina velutipes.

En la cumbre de la sierra de Carrascoy y en la parte alta de las de Espuña (p. ej. en el Valle de Leiva) y de Caravaca, nos encontramos en el área potencial del carrascal de Quercus rotundifolia, que persiste, más o menos disperso en el pinar actual. Alli se refugian numerosas especies de encinar, entre las que podemos citar Propolis versicolor, Calodon ferrugineum, Phellodon melaleucus, Phellinus torulosus, $\mathrm{Hy}$ grocybe conica, H. chlorophana, Clitocybe odora, Mycena pura, Amanita pantherina, Cortinarius ionochlorus.

Buen indicador del área potencial del encinar es Hygrophorus dichrous, que encontramos con extraordinaria abundancia y vigor en la sierra de Espuña, el 3/$\mathrm{XII} / 77$. Esta localidad marca probablemente el límite de esta especie tan apreciada en Cataluña y tan abundante en torno al eje Manresa-Igualada-Montblanc.

A mayor altitud aparecen bosques de Pinus pinaster, cuyas gruesas agujas presentan en el suelo las minúsculas ventanitas de Naemacyclus nize us, al tiempo que los grandes conos caidos son colonizados por Mycena seynii.

Mención aparte merecen las especies nitrófilas y eutróficas, especialmente abundantes en jardines y bordes de caminos, acequias, y huertos, entre las que dominan los Agaricus y Coprinus, junto a Psatbyrella candolleana, Agrocybe vervacti. Stropharia coronilla, Pholiota spumosa, Cyathus olla, especies particularmente oportunistas y a menudo efímeras.

En los barbechos y matorrales claros del interior, a una cierta altitud (p. ej. en la sierra de Caravaca) pueden hallarse buenas floraciones de Pleurotuseryngii, la seta de cardo, tan apreciada en el centro de España como escasa en los Países Catalanes.

Se echan en falta numerosas especies que una prospección más detenida y prolongada pondrá sin duda de manifiesto, permitiendo completar la visión muy general que tenemos ahora de la flora fúngica del S.E. de España. 


\section{ASCOMICETES}

\section{ESFERIALES}

\section{Hypomyces lateritius Fr.}

Sierra de Espuña. Valle de Leiva. Leg. Llimona, 3-12-77.

Parasitando Lactarius sanguifluus, sobre el que produce la típica tumefacción de formas, aumentando la densidad de la carne y provocando castración parasitaria, que conduce a la sustitución de las láminas por una superficie blanca, levemente arrugada.

Apiocraea chrysosperma (Tulasne) Sydow (forma conidica: Sepedonium chrysospermum (Bull.) Fr.)

San Pedro del Pinatar. Leg. Llimona, 5-11-77. s/Boletus, fluryi.

\section{FACIDIALES}

Lophodermium pinastri (Schr. ex Fr.) Cheval.

Venta de la Paloma. El Palmar. Leg. Llimona, 26-11-77. s/agujas de P. halepensis.

Naemacyclus niveus (Persoon ex Fries) Saccardo

Sierra de Caravaca. Cuerda de Robredo. Leg. Llimona, 31-10-77. s/aciculas muertas de Pinus pinaster:

\section{HELOCIALES}

Propolis versicolor (Fr.) Fr.

Sierra de Caravaca. Cortijo del Nevazo de Arriba. Cuerdą de Robredo. Leg. Llimona, 31-1077. s/madera. Ascos 8-esporados cilíndricos-fusiformes. Esporas hialinas, no septadas 19-25x $5-7 \mathrm{u}$.

Rutstroemia firma (Pers.) Karst

Sierra de Espuña. Valle de Leiva. Leg. Llimona, 3-12-77.

s/ramas muertas de P. halepensis. Abundante en el cauce seco del torrente.

\section{BASIDIOMICETES}

(Holobasidiomicetes)

\section{DACRIMICETALES}

Dacrymyces deliquescens (Bull.) Fr.

Sierra de Espuña. Entrada Valle de Leival. 1 km., arriba de la Escuela-Hogar. Legr. Llimona, 3-12-77. Abundante en todo el valle. Pinares P. halepensis con Q. corcifera, R. officinalis, P. lentiscus. 


\section{AFILOFORALES}

Ramaria stricta (Fr.) Quél., non Bourdot \& Galzin

Sierra de Espuña. Valle de Leiva. Leg. Llimona, 3-12-77.

s/madera muerta. Esporas de 7,5-8,5 x 3,1-4,6 u., elíptico-fusiformes.

Calodon ferrugineum Karst.

Sierra de Espuña. Fuente del Hilo, cerca del río Espuña, 850 m. Leg. F. Sánchez, A. Andrés Rubio, 7-11-77.

Phellodon melaleucus (Fr.) Karst.

= Calodon graveolens (Delast.) Quél.

Sierra de Espuña. Valle de Leiva. Leg. Llimona, 3-12-77. Pinares con Q. rotundifolia.

Hydnum repandum Fr.

Sierra de Espuña. Entrada Valle de Leiva. 1 km., arriba de la Escuela-Hogar. 700 metros. Leg. Llimona, 3-12-77.

Pinares $P$. halepensis con $Q$. coccifera, $R$. officinalis, $P$. lentiscus.

Stereum hirsutum (Fr.) S.F. Gray

Venta de la Paloma. El Palmar. Leg. Llimona, 26-11-77.

s/tocón de $P$. halepensis.

Sierra de Espuña. Entrada Valle de Leiva, Escuela-Hogar. Leg. Llimona 3-12-77. Pinar de $P$. halepensis con $Q$. coccifera.

Chondrostereum purpureum (Pers. ex Fr.) Pouz.

Sierra de Caravaca. Cortijo Nevazo de Arriba. Leg. Llimona, 31-10-77 Choperas plantadas.

Ceriporia bresadolae (Bdot. \& Galz.) Donk

= Poria bresadolae Bourdot et Galzin 1925

Sierra de la Fuensanta. El Valle. Leg. Llimona, 29-10-77.

Pinar de P. halepensis. s/madera muerta de P. halepensis. Esporas hialinas, lisas, cilindricas o débilmente curvadas $6,2-7,8 \times 1,5-23$, u.

Se trata de la misma especie encontrada por Malençon y Llimona en el Desierto de Las Palmas el 24-10-77 y que nosotros hemos vuelto a encontrar numerosas veces en primavera, tanto en Sierra de La Fuensanta como en Sierra Espuña y El Carche.

Bjerkandera adusta (Willd. ex Fr.) Karst.

= Leptoporus adustus (Willd.) Quél.

Murcia. Parque del Malecón. Leg. P. del Olmo, M. Hurtado, 4-11-77. Jardin. s/madera muerta.

Inonotus hispidus (Bull. ex Fr.) Karst.

Almansa. Santuario de Belén. Leg. Honrubia, 5-11-77.

s/ Populus hvbrida, cerca de una acequia.

Alhama de Murcia. Leg. A. Martinez, 5-12-77. Muy abundante sobre viejos choposen toda la región murcianat. 
Phellinus pini (Thor. ex Fr.), A. Pilat 1941.

= Xanthochrous pini Patouillard 1900

Almansa. Zona del Pantano. Leg. Honrubia, 5-11-77. Pinares s/P. halepensis. Muy abundante.

Aceniche (SW de Bullas) Leg. Egea, 6-11-77. Pinares de P. pinea, con P. lentiscus. Cistus albidus y $Q$. coccifera.

Phellinus pomaceus (Pers. ex S.F. Gray) Maire

Totana. Leg. A. Martínez, 27-11-77. s/almendro. Aspecto de pequeño Ph. igniarius (L. ex Fr.) Quél. Esporas 5,5-6,5 x 4-5 u., elipticas.

Phellinus torulosus (Pers.) Bdot. \& Galz.

Almansa. Santuario de Belén. Leg. Honrubia, 5-11-77. En grupos, s/olmo, cerca de una acequia.

Fomitopsis pinicola (Sow ex Fr.) Karst.

Sierra de Espuña. Valle del río Espuña. Leg. Llimona, 9-10-77.

Sierra de Espuña. Entrada Valle de Leiva. Escuela-Hogar. Leg. Llimona, 3-12-77. Pinares de $P$. halepensis con $Q$. coccifera, $R$. officinalis, $P$. lentiscus. Abundante s/tocones de $P$. halepensis. Muy abundante en los pinares de las montañas murcianas.

Leucoporus arcularius (Bats.) Quél.

Aceniche (SW de Bullas). Leg. Egea, 6-11-77. Pinares de P. pinea con P. lentiscus, Cistus albidus, $Q$. coccifera, s/madera muerta.

Esporas 8-9,3 x 3,5-4,6u., cilindrico-subfusiformes.

Boletopsis leucomelas (Pers. ex Pers.) Fay.

= Polyporis leucomelas Pers.

= B. subsquamosa (L. ex Fr.) Kotl \& Pomz.

Sierra de Espuña. La Perdiz. Leg. P. Cano, M. Hurtado, P. del Olmo, 6-11-77. Bosque de $P$. halepensis. Esporas 3,9-5,4 u., verrucosas, esféricas.

\section{Pleurotus cornucopiodes Fr.}

=Pl. cornucopiae Paul. ex Fr.

Sierra de Caravaca. Leg. Llimona, 31-10-77. Bosque de Populus; lignicola, 3,5 cm., diámetro, Pie $11 \mathrm{~mm}$., altura; olor farinoso. Esporas. 7-8,5 x 4-5 u., cilindricas.

Pleurotus eryngii (D.C. ex Fr.) Quél.

Sierra de Caravaca. Cortijo Nevazo de Arriba, cuerda de Robredo. Leg. Llimona, 31-10-77. Campo abandonado con Helychrysum stoechas y Eryngium campestre. Abundante. Esporas 9,7-11,7 x 4,6-5,4 u., eliptico-cilindricas.

Sierra de Espuña. El Mugrón Grande. Leg. P. Rodriguez y F. Ruiz Vendrell, 27-11-77. P. pinaster.

Sierra de Espuña. Entrada Valle de Leiva. Escuela Hogar. Leg. Llimona, 3-12-77. Pinares de $P$. halepensis con $Q$. coccifera. $R$. officinalis, $P$. lentiscus. 
Pleurotus ostreatus (Jacq. ex Fr.) Kummer

Sierra de Caravaca. Cortijo Nevazo de Arriba. Leg. Llimona, 31-10-77. Choperas plantadas, s/tronco de chopo.

Sierra de Espuña. Entrada Valle de Leiva. Escuela-Hogar. Leg. Llimona, 3-12-77. Pinares de $P$. halepensis con $Q$. coccifera, $R$. officinalis, $P$. lentiscus.

Alhama de Murcia. Leg. A. Martínez, 5-12-77. s/tronco.

\section{Schizophyllum commune Fr.}

Sierra de Moratalla. Cerca del río Benamor. Leg. Rosa Verdú et al., 23-10-77. s/P. halepensis.

Sierra de Caravaca. Cortijo Nevazo de Arriba. Leg. Llimona, 31-10-77. Chopera plantada s/ Populus sp.

Murcia. Parque del Malecón. Leg. P. del Olmo, M. Hurtado, 4-10-77.

Sierra de Espuña. El Mugrón Grande, Leg. P. Rodríguez. F. Ruiz. 27-11-77. P. pinaster. Muy abundante.

Sierra de Espuña. Valle de Leiva. Escuela-Hogar. Leg. Llimona, 3-12-77. s/P. halepensis. Pinar de $P$. halepensis con $Q$. coccifera, $R$. officinalis, $P$. lentiscus.

\section{BOLETALES}

Suillus bellinii Inz.

Sierra de Espuña, cerca del Refugio. Pequeño valle. Leg. Llimona, 9-10-77. Esporas 9,3-11 x 3,9-5 u., elipticas.

Sierra de Caravaca. Cortijo Nevazo de Arriba. Leg. Llimona 31-10-77.

Cartagena. Falda del Roldán. Parque de Tentegorra. Leg. F. Pérez Aguilar, M. Martíne Velasco, 6-11-77. Pinares de P. halepensis.

Sierra de Ricote. Leg. R. Guillamón, 6-11-77. Pinar de P. halepensis.

Sierra de Espuña. Fuente del Hilo. $850 \mathrm{mts}$. Leg. F. Sánchez, A. Andreo, 7-11-77.

Sierra de la Fuensanta. El Valle. Leg. M. Cano, M. Hurtado, P. del Olmo, 6-11-77. Pinar P. halepensis.

\section{Suillus fluryi Huijs.}

Sierra de los Cuchillos. Bullas. Leg. Egea 2-11-77. Pinar P. halepensis con Q. coccifera. Esporas 8-9,3 x 4-5,4 u., Pie más bien bajo, cutícula viscosa, separable, pardo-oliváceo, con aspecto radialmente estriado. Carne blanda, color amarillo pálido, amarillo intenso bajo los poros. Sabor agradable, olor fragante.

Sierra de Caravaca, Cortijo Nevazo de Arriba. Leg. Llimona, 31-10-77. Campos abandonados en proceso de recolonización.

Sierra de la Bureta. Bullas. Leg. Egea, 3-11-77.

Paseo del Malecón. Murcia. Leg. P. del Olmo, M. Hurtado, 4-11-77.

Almansa. Zona del Pantano. Leg. Honrubia, 5-11-77. Pinares de P. halepensis.

La Puerta. Moratalla. Margen derecho del río Benamor. Leg. Rosa Verdú, 5-11-77. Caliza. $P$. clusiana y $P$. halepersis.

Aceniche (SW de Bullas) Leg. Egea, 6-11-77. Pinares P. pinea, con P. lentiscus, Cistus albidus, $Q$. coccifera.

Sierra de Ricote. Leg. R. Guillamón, 6-11-77. Pinar P. halepensis.

Cartagena. Falda del Roldán. Parque de Tentegorra. Leg. F. Pérez Aguilar, M. Martínez, 611-77. Pinar. P. halepensis.

Sierra de Espuña. Fuente del Hilo. 850 mts., Leg. F. Sánchez, A. Andreo, 7-11-77. Pinares de P. halepensis. 
Sierra de la Fuensanta. El Valle. Leg. P. J. Lax, 8-11-77.

Sierra de La Fuensanta. Cerca de la urbanización de la Cresta del Gallo. Leg. M. I. Molına, 20-11-77. Pinar. P. halepensis.

Sierra de la Fuensanta, de El Castillo hacia El Cerrillar. Leg. F. Barba, 27-11-77. Pinares. $P$. halepensis.

Es sin duda la especie más abundante en los pinares térmicos y secos de la región murciana, desde las dunas de San Pedro del Pinatar hasta los bosques altos de la Sierra de Caravaca, y en este último caso, en la solana. Después de las lluvias puede aparecer en enorme número y alcanzar tamaños desmesuradamente grandes.

\section{Paxillus panuoides Fr.}

Sierra de Espuña. Valle de Leiva. Leg. Llimona, 3-12-77.

Alhama de Murcia. Leg. A. Martínez, 5-12-77.

Chroogomphus rutilus (Schff. ex Fr.) O. K. Miller

$=$ Gomphidius viscidus L. ex Fr.

Sierra de Caravaca. Cortijo Nevazo de Arriba. Leg. Llimona 31-10-77. Abundante.

Sierra de La Lavia, cerca de Bullas. Leg. Egea, 1-11-77.

Sierra de los Cuchillos, cerca de Bullas. Leg. Egea, 2-11-77.

Cartagena. Falda del Roldán. Parque de Tentegorra. Leg. F. Pérez Aguilar, M. Martínez Velasco, 6-11-77. Pinar. P. halepensis.

Sierra de Espuña. Entrada Valle de Leiva. Escuela-Hogar. 700 m. Leg. Llimona, 3-12-77. Pinares de P. halepensis con Q. coccifera, R. officinalis, P. lentiscus. Muy abundante. Asociado a $S$. fluryi.

\section{AGARICALES}

Hygrophorus chlorophanus Fr.

Entrada Valle de Leiva. Escuela-Hogar. Sierra de Espuña. Leg. Llimona, 3-12-77. Pinares $P$. halepensis con $Q$. coccifera, $R$. officinalis. P. lentiscus.

Hygrophorus dichrous Kühn. et Romagn.

Sierra de Espuña. Entrada Valle de Leiva. Escuela-Hogar. Leg. Llimona, 3-12-77. Muy abundante por rodales. Pinares de $P$. halepensis con $Q$. coccifera, $R$. officinalis, P. lentiscus. Excelente comestible, pero no buscado por los micófagos locales.

Camarophyllus carneogriseus (G. Malençon) comb. nov. (1)

Collado s/Cortijo Nevazo de Arriba. Sierra de Caravaca. Calar de Ortega. Leg. Llimona, 31-10-77. s/Margas con J. oxycedrus y Genista scorpius. Especie relativamente frecuente en pinares termófilos mediterráneos a partir de la provincia de Tarragona. Esporas subovales, algo reniformes 7,8-8,5 x 4,6-5,4 u., pared lisa y delgada. Sombrero $2 \mathrm{cms}$, de diámetro extendido, centralmente deprimido. Cutícula carneogrisácea, seca, con fisuras concéntricas. Margen largo tiempo enrollado, Pie $35 \times 7 \mathrm{~mm}$., cilindrico, algo claviforme hacia la base, con rizomorfas basales; cutícula carneogrisácea. Láminas rosadas cárneas. Carne blanca. Olor a harina intenso.

Hygrocybe conica (Scop. ex Fr.) Kummer

Sierra de Caravaca. Cortijo Nevazo. Leg. Llimona 31-10-77 Pinar de P. halepensis. 
HONGOS DEL S.E. DE ESPAÑA. I.

Sierra de Espuña. Entrada Valle de Leiva. Escuela-Hogar. Leg. Llimona, 3-12-77. Pinares de $P$. halepensis con $Q$. coccifera. $R$. officinalis y $P$. lentiscus.

Clitocybe dealbata (Sow. ex Fr.) Kummer

Sierra de Espuña. Valle de Leiva. Escuela-Hogar. Leg. Llimona, El Cañarico. Murcia. Leg. E. Molero, 3-12-77.

Alhama de Murcia. Leg. A. Martínez, 5-12-77.

San Pedro del Pinatar. Leg. Llimona, 5-11-77. Dunas con P. halepensis. 1,5-2,1 cms. de diá. metro. Blanco ligeramente alutáceo. Esporas elípticas 4-5 x 3,1 u.; pared delgada, lisa.

Clitocybe gibba (Pers. ex Fr.) Kumm.

= Clitocybe infundibuliformis (Schff. ex Fr.) Quél.

El Palmar. Venta de la Paloma, hacia el Cigarrón. Leg. M. ${ }^{a}$ J. Quiles, M. ${ }^{a}$ J. Martínez, F. Ruiz y P. Rodriguez, 5-11-77.

Clitocybe odora (Bull. ex Fr.) Kummer

Sierra de La Fuensanta. De El Castillo hacia el Cerrillar.

Leg. F. Barba, 27-11-77. Pinares de P. halepensis.

Tricholoma caligatum (Viv.) Rick.

Sierra de Espuña. La Perdiz. Leg. Llimona, 6-11-77. Pinares de P. halepensis. Esporas 5,46,5 x 4,5-6 u.

Sierra de Espuña. El Mugrón Grande. Leg. P. Rodríguez y F. Ruíz, 27-11-77. Pinares de $P$. pinaster. Olor característico de perfume oriental, que recuerda un poco el de la canela.

Tricholoma populinum Lange

Sierra de la Lavia, cerca de Bullas. Leg. Egea, 1-11-77.

Tricholoma psammopus (Kalch.) Quélet

Alhama de Murcia. Leg. A. Martínez, 5-12-77. Pinares.

Sierra de los Cuchillos, cerca de Bullas. Leg. Egea 2-11-77. Pinares de P. halepensis con $Q$. coccifera.

Sierra de Espuña, entrada Valle de Leiva, Escuela-Hogar. 700 m. Leg. Llimona, 3-12-77.

Pinares de $P$. halepensis con $Q$. coccifera.

Tricholoma terreum (Schaeff. ex Fr.) Kummer

Sierra de los Cuchillos, cerca de Bullas. Leg. Egea, 2-11-77. Pinares de P. halepensis con $Q$. coccifera.

Sierra de Espuña. El Mugrón Grande. Leg. P. Rodríguez, R. Ruíz 27-11-77. Pinares de $P$. pinaster.

Sierra de Espuña. Entrada Valle de Leiva. Escuela-Hogar. 700 m. Leg. Llimona, 3-12-77

Abundante en pinares de $P$. halepensis.

Tricholoma ustale (Fr. ex Fr.) Kummer

Sierra de Espuña. Valle de Leiva. Escuela-Hogar. Leg. Llimona 3-12-77. Pinares de Q. coccifera, $R$. officinalis, P. lentiscus, Qu. rotundifolia. 


\section{Tricholoma ustaloides Romagn.}

= Tricholoma albobrunneum (Pers. ex Fr.) Kumm. ss. Malençon

Aceniche (SW de Bullas). Leg. Egea 6-11-77. Pinares de P. pinea con P. lentiscus, Cistus albidus, $Q$. coccifera.

Sierra de Espuña. Entrada Valle de Leiva. Escuela-Hogar. 700 m. Leg. Llimona 3-12-77. Abundante en pinares de $P$. halepensis, con $Q$. coccifera, $R$. officinalis, $P$. lentiscus.

Melanoleuca grammopodia (Bull. ex Fr.) Pat.

Paseo del Malecón. Murcia. Leg. P. del Olmo, M. Hurtado, 4-11-77.

Sierra de Espuña. Orillas del río Espuña. F. Sánchez. A. Andrés, 7-11-77. Pinares de P. halepensis, orillas del río. Esporas 8,5-10 x 5,4-6,2 u., oval-redondeadas.

\section{Collybia dryophila Kumm.}

= Marasmius dryophilus (Bull. ex Fr.) Karst.

Sierra de la Bureta. Bullas. Leg. Egea, 3-11-77. P. halepensis.

Sierra de los Cuchillos. Bullas. Leg. Egea, 2-11-77. Pinares.

Sierra de Espuña. Fuente del Hilo. 850 m. Leg. F. Sánchez, A. Andrés 7-11-77. Pinares. P. halepensis.

Sierra de La Fuensanta. El Valle. Leg. Llimona, 29-10-77. Cerca de P. halepensis.

Sierra de Caravaca. Cortijo Nevazo de Arriba. Leg. Llimona, 31-10-77. Rastrojos.

Sierra de Espuña. El Mugrón Grande. Leg. P. Rodríguez, F. Ruiz. Vendrell, 27-11-77. Pinar de $P$. pinaster.

Collybia impudica (Fr.) Singer

= Marasmius impudicus Fr.

Sierra de La Fuensanta. El Valle. Leg. Llimona, 29-10-77. Suelo pedregoso, siliceo, con $P$. halepensis. Esporas 6,2-7,5 x3,1-3,9u., elíptico-larmiformes. Sombrero pardorrojizo $2,5 \mathrm{~cm}$ de diámetro. Pie pardo-grisáceo. Típico olor a col o mercaptanos.

Mycena pura (Pers. ex Fr.) Kumm.

Sierra de los Cuchillos, cerca de Bullas. Leg. Egea, 2-11-77. Abundante, en pinares de $P$. halepensis con $Q$. coccifera.

Mycena seynii Quél.

Sierra de Caravaca. Cortijo Nevazo de Arriba. Leg. Llimona 31-10-77. s/conos de P. halepensis.

Sierra de la Bureta, cerca de Bullas. Leg. Egea 3-11-77, pinares de P. halepensis.

Sierra de Espuña. Entrada Valle de Leiva, Escuela-Hogar. Leg. Llimona 3-12-77. Abundante en pinares de $P$. halepensis con $Q$. coccifera, $R$. officinalis, $P$. lentiscus.

Fammulina velutipes (Curt. ex Fr.) Karst.

= Collybia velutipes Quél.

Sierra de Caravaca. Cortijo Nevazo de Arriba. Leg. Llimona 31-10-77, lignícola, s/tocón de Populus sp. Esporas 7,8-9,5 $\times$ 4-5 u., cilindricas, pared delgada, lisa.

Entoloma (Nolanea) clandestinum (Fr.)

Sierra de Caravaca. Leg. Llimona 31-10-77. Encinar. 
Entoloma (Eccilia) rusticoides (Gill.) Lang.

$=$ Rh. tristis Bres.

Venta de la Paloma. El Palmar. Leg. M. J. Quiles 5-11-77. En suelo compacto, arcilloso, cubierto de musgos ralos, en el pinar de P. halepensis.

Aspecto de Omphalina. Esporas poligonales, globulosas, isodiamétricas 7,8*9,3 x 7,8 u. La nuestra coincide bastante bien con la descripción de Eccilia tristis Bres., hecha por Malençon y Bertault (1970) sobre ejemplares del Atlas Medio.

Amanita ovoidea (Bull. ex Fr.) Quél.

La Puerta. Moratalla. Margen derecho del río Benamor. Leg. R. Verdú 5-11-77. Calizas. $P$. clusiana y $P$. halepensis.

El Palmar. Venta de la Paloma. Leg. M. J J. Quiles, M. J. Martínez, F. Ruiz y P. Rodriguez, 5-11-77. Bosque de P. halepensis.

Sierta' de La Fuensanta, cerca de la urbanización de la Cresta del Gallo. Leg. M. I. Molina 20-11-77. En pinares de P. halepensis.

Sierra de Espuña. El Mugrón Grande. Leg. P. Rodriguez, F. Ruiz Vendrell, 27-11-77. Pinar de $P$. pinaster.

Sierra de Espuña. Entrada Valle de Leiva. Escuela-Hogar, Leg. Llimona 3-12-77. Abundante en los lugares mas soleados del pinar de $P$. halepensis.

Amanita pantherina Fr. ex D.C.

Sierra de Espuña. Entrada Valle de Leiva. Escuela-Hogar. Leg. Llimona 3-12-77. Pinares de $P$. halepensis con $Q$. coccifera, $R$. officinalis, $P$. lentiscus. Tres o cuatrocarpóforos en un sólo micelio.

Agaricus meleagris Schaeff.

La Alberca. Sierra de La Fuensanta. Leg. G. Guzmán 3-11-77. Bajo eucalipto. Sombrero $10,5 \mathrm{~cm}$ de diámetro, convexo, algo deprimido; cutícula con escuámulas fibrilosas, triangulares, parduzcas. Pie $9 \times 3,2 \mathrm{~cm}$ Color blanco liso. Anillo de aspecto doble. Carne dura, color inmutable o amarillea al tacto en el sombrero; también amarillea (citrino vivo) en el pie, por presión. Olor a almendras amargas. Esporas 5,4-6,5 x 3,5-4,6 u.

Agaricus silvicola (Vitt.) Sacc.

Sierra de la Lavia, cerca de Bullas. Leg. Egea, 1-11-77.

Olor ligeramente anisado. Sombrero $6 \mathrm{~cm}$. de diámetro. Pie fistuloso, pardusco en la base, $67 \times 16 \mathrm{~mm}$; cutícula blanca que amarillea al tacto. Anillo descendente amplio. Sombrero con enrojecimiento ligero.

Agaricus xanthoderma Gen.

Paseo del Malecón. Murcia. Leg. P. del Olmo, M. Hurtado, 4-11-77. Tierra de jardín.

Macrolepiota mastoidea (Fr.) Sing.

Sierra de Espuña. Fuente del Hilo. 850 m. Leg. F. Sánchez, A. Andrés, 7-11-77. Pinares, orillas del río.

Coprinus atramentarius Bull. ex Fr.

Parque del Malecón. Murcia. Leg. P. del Olmo, M. Hurtado, 4-11-77. Jardín. Sombrero 4 
$\mathrm{cm}$, de diámetro x 4 de altura; cutícula viscosa. Pie $75 \times 9 \mathrm{~mm}$, (base), fistuloso, blanco. Esporas 9,3-11,7 x 5,4-6,2 u., elípticas.

Coprinus micaceus (Bull. ex Fr.) Fr.

El Cañarico. Murcia. Leg. E. Molero, 3-12-77.

Psathyrella (Hypholoma) candolleana (Fr.) Mre.

Sierra de Caravaca. Cortijo Nevazo de Arriba. Leg. Llimona, 31-10-77. Choperas plantadas, al borde del camino.

Agrocybe aegerita (Brig) Sing.

Almansa. Santuario de Belén. Leg. Honrubia, 5-11-77. s/Populus sp., cerca de una acequia. Esporas 8,5-10,5 x 5,4-6,2 u., elipticas, débilmente amigdaliformes.

Agrocybe vervacti (Fr.) Romagn.

Parque de las Palmeras. Murcia. Leg. Llimona 15-1-78 s/suelo arenoso, desnudo, numerosos ejemplares, densamente cespitosos.

Stropharia coronilla (Bull. ex Fr.) Quél.

Almansa. Santuario de Belén. Leg. Honrubia, 5-11-77. Cerca de una acequia con gramíneas, bajo olmos. Sombrero $4-5 \mathrm{~cm}$ de diámetro convexo, ocre claro pajizo; cutícula viscosa. Pie 40-45 x 7-10 mm, blanco que amarillea al tacto. Anillo estriado. Láminas pardo oscuro violáceo. Carne de olor desagradable, metálico ligero. Esporas 8,5-10 x 4,6-6 u., elíptico-ovales. Basidios cilíndricos mazudos. Cistidios claviformes no muy abundantes, sin cristales aparentemente.

Pholiota (Flammula) spumosa (Fr.) Sing.

Sierra de Espuña. El Mugrón Grande. Leg. P. Rodriguez, F. Ruiz, 21-11-77. Bosque P.pinaster. Sombrero, disco pardorrojizo, borde amarillo claro, carne amarillo claro. Pie citrino, con mechas rojas; sin anillo. Esporas 6-8 x 4-4,6 u., elipticas, pruniformes. Pleurocistitios ventrudos, sin cristales.

Inocybe caesariata (Fr.) ss. Heim

San Pedro del Pinatar. Leg. Llimona, 5-11-77. Dunas con P. halepensis, fuera del bosque. Abundante. Sombrero 2,2-3-3,8 cm de diámetro. Pie 2-3 cm x 7-8 mm. Cortina visible. Olor agradable. Leiosporados. Esporas 9,3-11,7 x 4,5-6,2 u., elípticas. Queilocistidios ventrudos, siempre sin cristales; muy abundantes.

El nuestro, puede representar una forma de tránsito semejante al $l$. caesariata (Fr.) ss. Heim citado por J. M. Losa Quintana (1960) en las dunas de Castelldefels, donde es muy abundante; probablemente incluíble dentro del I. dulcamara (A. \& S. ex Pers.) Kummer, descrito en Malençon y Bertault (1970), dado que ambas especies de dunas no parecen estar claramente diferenciadas.

Inocybe fastigiata (Schff. ex Fr.) Quél.

Sierra de la Fuensanta, cerca de la urbanización de la Cresta del Gallo. Leg. M. I. Molina 2011-77. 
Inocybe geophylla (Sow. ex Fr.) Kummer

Sierra de Espuña. Valle de Leiva. Leg. F. Sánchez 3-12-77.

Sierra de Espuña. Entrada Valle de Leiva. Escuela-Hogar. Leg. Llimona 3-12-77. Pinares de $P$. halepensis con $Q$. coccifera, $R$. officinalis, $P$. lentiscus.

\section{Hebeloma edurum Métrod}

Sierra de Espuña. Valle de Leiva. 3-11-77. Pinar. P. halepensis.

Sierra de Espuña. Fuente del Hilo. Leg. F. Sánchez, 7-11-77.

Sierra de la Fuensanta. De El Castillo hacia El Cerrillar. Leg. F. Barba 27-11-77. Pinar de $P$. halepensis.

Hebeloma mesophaeum (Pers. ex Fr.) Quél.

Sierra de Caravaca. Cortijo Nevazo de Arriba. Leg. Llimona 31-10-77. Bajo encinar. Olor rafanoide muy débil. Sombrero 2,4-2,7-3,2 cm de diámetro, alutáceo. Pie con cortina, 45-50 x 6$7 \mathrm{~mm}$. Esporas 7,8-10,9 x 5,4-6,2 u., elípticas.

Cortinarius (Phlegmacium) ionochlorus R. Maire

Sierra de Espuña. El Mugrón Grande. Leg. P. Rodríguez 27-11-77. Encinar de Q. rotundifolia con $P$. pinaster.

\section{RUSULALES}

Russula sanguinea (Bull. ex St. Am.) Fr.

Aceniche (SW de Bullas). Leg. Egea 6-11-77. Pinares de P. pinea con P. lentiscus, Cistus albidus, Q. coccifera.

Russula torulosa Bres.

Sierra de La Fuensanta. De El Castillo a El Cerrillar. Leg. F. Barba 27-11-77. Pinares de $P$. halepensis. Junto a Hebeloma edurum. Esporas 7,5-10 x 6,2-7,8 u. Redondeadas, elipticas.

Lactarius deliciosus Fr.

Sierra de los Cuchillos, cerca de Bullas. Leg. Egea 2-11-77. Pinares con P. halepensis y $Q$. coccifera.

Sierra de Espuña. Entrada Valle de Leiva. Escuela-Hogar. Leg. Llimona 3-12-77. Pinares de $P$. halepensis con $Q$. coccifera.

Lactarius sanguifluus (Paul. ex Fr.) Fr.

Aceniche (SW de Bullas). Leg. Egea 6-11-77. Pinar de $P$. pinea.

Sierra de Espuña. Entrada Valle de Leiva. Escuela-Hogar. Leg. Llimona 3-12-77. Pinar de $P$. halepensis con $Q$. coccifera.

Ambas especies, abundantes y muy buscadas en noviembre/diciembre, reciben el nombre de "guiscanos". 


\section{GASTERALES}

Geastrum pseudostriatum Hollós

Aceniche (SW de Bullas) Leg. Egea, 6-11-77. Pinares de P. pinea con P. lentiscus, Cistus albidus, $Q$. coccifera.

\section{Rhizopogon rubescens Tulasne}

Sierra de La Fuensanta. El Valle. Leg. M. Hurtado, P. del Olmo, 6-11-77. Pinares de P.halepensis.

Cyathus olla Batsch trans Pers.

Sierra de La Fuensanta. El Valle. Leg. Llimona, 29-10-77. Bosque de P. halepensis, al borde del camino.

Sierra de Espuña. Entrada Valle de Leiva. Escuela-Hogar. Leg. Llimona 3-12-77. Pinares de $P$. halepensis con Q. rotundifolia, $R$. officinalis, P. lentiscus.

\section{BIBLIOGRAFIA}

BOURDOT, H. et A. GALZIN, Hyménomycètes de France. Soc. Mycol. de France. Lechevalier. Paris. $761 \mathrm{pp}$.

CALONGE, F. D.; 1975. Hongos de nuestros campos y bosques. ICONA. Madrid. 1-343 pp.

DENNIS, R.W.G., 1968. British Ascomycetes. Cramer Verlag. Stuttgart. 1-434 pp.

HEIM, R., 1934. Observations sur la Flore Mycologique Catalane. Publ. Mus. de Ciènc. Nat. de Barcelona. 15 (3 ser.) n. ${ }^{\circ} 3$.

- 1969. Champignons d'Europe. Boubée. Paris. 1-680 pp.

KÜHNER, R. et H. ROMAGNESI, 1974. Flore analytique des champignons supérieurs. Paris. 556 pp.

LANGE, J. E., D. M. LANGE, X. LlimONA, 1976. Guía de campo de los hongos de Europa. Omega. Barcelona. 291 pp.

LOSA QUINTANA, J. M., 1960. Contribución al estudio y revisión de los Inocybes españoles. Inst. A.J. Cavanilles. Barcelona.

MAIRE, R., 1933. Contributions à l'étude de la Flore Mycologique de la Catalogne. Publ. Mus. de Ciènc. Nat. de Barcelona. 15. n. ${ }^{\circ} 2.1-120$ pp.

- 1937. Contribution à l'étude de la Flore Mycologique de la Catalogne. Publ. de l'Inst. Bot. de Barcelona. 3 n. ${ }^{\circ} 4$.

MALENÇON, G. et R. BERTAULT, 1970, 1975. Flore des champignons supérieurs du Maroc. Tomes I, II. Rabat.

- 1971. Champignons de la Péninsule Ibérique. III Acta Phytotax. Barcinonensia 8: 1-97.

- 1972. Champignons de la Péninsule Ibérique. IV Acta Phytotax. Barcinonensia 11: 161.

- 1976. Champignons de la Péninsule Ibérique. V Acta Phytotax. Barcinonensia 19: I68. 
MARCHAND, A., 1971, 1973, 1974, 1976. Champignons du nord et du midi. Soc. Mycol. des Pyrénées Méditerranéennes. Perpignan. Tomes 1, 2, 3, 4.

MÜLLER, E. y W. LOEFFLER, 1976. Micología. Omega. Barcelona.

MOSER, M. 1963. Ascomyceten, in Kleine Kryptogamenflora. Band II a. G. Fischer Verlag. Stuttgart. 1-150 pp.

- 1978. Die Röhrlinge und Blätterpilze, in Kleine Kryptogamenflora. Bd. II b/2. G. Fischer Verlag. Stuttgart. 1-532 pp.

ROMAGNESI, H., 1963, 1970, 1971, Petit atlas des champignons. Bordas. Paris. 